\title{
Prosthetic Valve Dysfunction 35 Years after Mitral Valve Replacement with a Starr-Edwards Caged-disc Valve
}

\author{
Tetsuro Yokokawa ${ }^{1}$, Takahiro Ohara ${ }^{1}$, Seiji Takashio $^{1}$, Mari Sakamoto ${ }^{1}$, Yuko Wada ${ }^{1}$, \\ Kenji Nakamura ${ }^{1}$, Hiroyuki Takahama ${ }^{1}$, Makoto Amaki ${ }^{1}$, Takuya Hasegawa ${ }^{1}$, \\ Yasuo Sugano ${ }^{1}$, Hideaki Kanzaki ${ }^{1}$, Satoshi Yasuda ${ }^{1}$, Hisao Ogawa ${ }^{1}$, Tomoyuki Fujita ${ }^{2}$, \\ Junjiro Kobayashi ${ }^{2}$, Yoko Okamoto ${ }^{3}$, Taka-aki Matsuyama ${ }^{3}$, \\ Hatsue Ishibashi-Ueda ${ }^{3}$ and Toshihisa Anzai ${ }^{1}$
}

\begin{abstract}
A 49-year-old man was admitted to our hospital with a chief complaint of dyspnea. He had a history of mitral valve replacement (MVR) with a Starr-Edwards (SE) caged-disc valve at the age of 14. Echocardiography revealed elevated trans-valvular pressure gradient of the mitral prosthetic valve with neither disk motion abnormality nor abnormal structure. Catheterization confirmed an elevation of the mean diastolic gradient of the mitral valve to $12.3 \mathrm{mmHg}$. Re-MVR was performed, and abnormal tissue attached to the cage of the valve and proliferating beneath the valve was observed. Histologic examination revealed them as fibrinous tissue and mild pannus proliferation, respectively. This rare case report focuses on long-term follow-up and the complication of a SE caged-disc valve. A SE caged-disc valve may become stenotic, only detected with a trans-valvular pressure gradient without any disk motion abnormality or abnormal structure during a prolonged follow-up period.
\end{abstract}

Key words: mitral valve replacement, prosthetic valve dysfunction, Starr-Edwards caged-disc valve, pannus, fibrinous tissue

(Intern Med 55: 479-483, 2016)

(DOI: 10.2169/internalmedicine.55.5466)

\section{Introduction}

The Starr-Edwards (SE) caged-disc valve, model 6520, was developed in the early 1970 s for mitral valve replacement (MVR). The SE caged-disc valve was used for only a short period of time before being discontinued universally. Some patients lived with the SE caged-disc valve for a long time. The very long-term (over 30 years) outcome of the SE caged-disc valve has been reported to be associated with structural deterioration in a few patients (1). We witnessed a case of SE caged-disc valve dysfunction due to fibrinous tissue attached to the cage and mild pannus formation 35 years after MVR, in which the anatomical diagnosis was preoperatively difficult to make.

\section{Case Report}

A 49-year-old man was admitted to our hospital complaining of dyspnea. He had a history of cerebral infarction due to infective endocarditis, and MVR with a SE cageddisc valve model 6520 for mitral regurgitation and infective endocarditis in our hospital at 14 years of age. Twelve years after the MVR procedure, paroxysmal atrial fibrillation occurred. At 31 years of age, the patient underwent catheter ablation for atrial flutter. Thirty-five years after the MVR, when the patient was 49 years old, he suffered from leg edema and dyspnea on effort.

Transthoracic echocardiography revealed an elevated mean trans-valvular pressure gradient of $10.8 \mathrm{mmHg}$, with

${ }^{1}$ Department of Cardiovascular Medicine, National Cerebral and Cardiovascular Center, Japan, ${ }^{2}$ Department of Cardiovascular Surgery, National Cerebral and Cardiovascular Center, Japan and ${ }^{3}$ Department of Pathology, National Cerebral and Cardiovascular Center, Japan

Received for publication March 27, 2015; Accepted for publication June 1, 2015

Correspondence to Dr. Takahiro Ohara, tkohara@ncvc.go.jp 

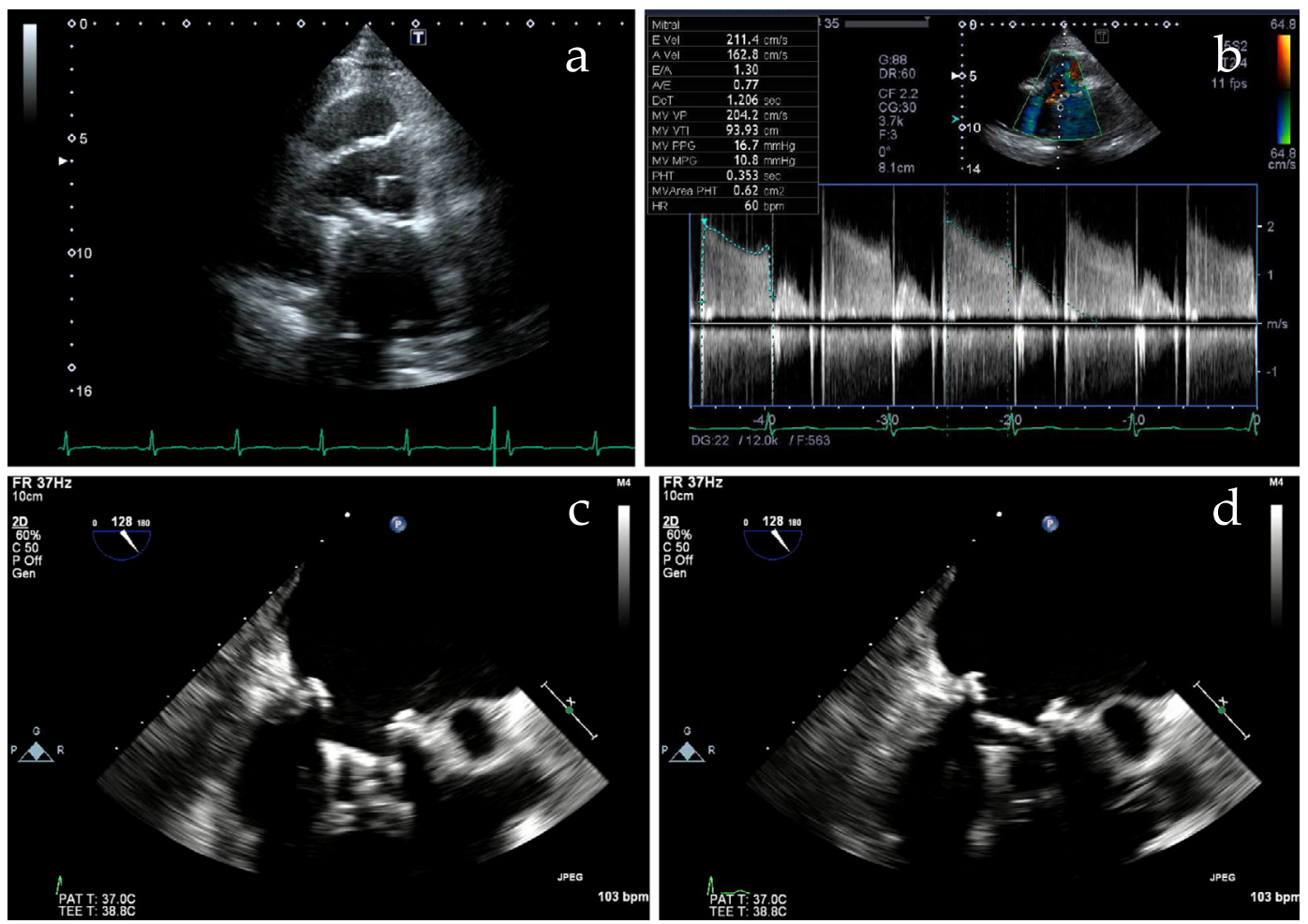

Figure 1. Two-dimensional transthoracic (a, b) and transesophageal (c, d) echocardiography. a: Transthoracic echocardiography did not indicate either any significant motion or structural abnormality of the disc. b: A Doppler continuous flow revealed an elevated mean trans-valvular pressure gradient of $10.8 \mathrm{mmHg}$. The peak velocity was $2.0 \mathrm{~m} / \mathrm{s}$ and the pressure half time was $353 \mathrm{msec}$. $\mathrm{c}$, $\mathrm{d}$ : Two-dimensional transesophageal echocardiography did not reveal any abnormalities that would cause mitral dysfunction (c: diastole, d: systole).
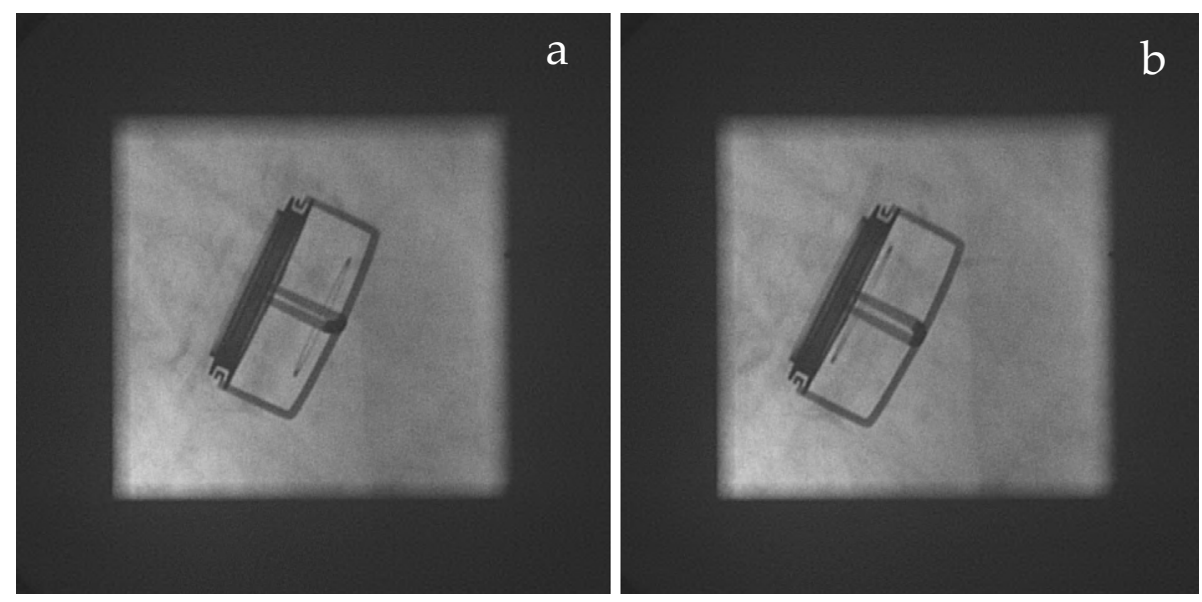

Figure 2. Cineradiography of the mitral prosthetic valve showing no restrictive motion of the disc. a: Diastolic phase. b: Systolic phase.

neither disc motion abnormality nor significant abnormal structure (Fig. 1). Electrocardiography displayed atrial fibrillation. Cardiac catheterization showed a mean pressure difference between the pulmonary capillary wedge pressure and left ventricular pressure of $12.3 \mathrm{mmHg}$. The mean pulmonary artery pressure was $24 \mathrm{mmHg}$ and cardiac index was $2.26 \mathrm{~L} / \mathrm{min} / \mathrm{m}^{2}$. Cineradiography of the mitral valve indicated that the disc could still move (Fig. 2). Transesophageal echocardiography did not find relevant structural abnormality regarding the mitral valve (Fig. 1, 3). Although we did not find any structural abnormality that would explain the dysfunction, we diagnosed the patient as having symptomatic prosthetic mitral valve stenosis of the SE caged-disc valve.

He underwent MVR with a St. Jude Medical mechanical valve and maze procedure. Intraoperative observation showed mild pannus formation on the atrial side of the prosthetic valve and abnormal tissue attaching to the cage of 

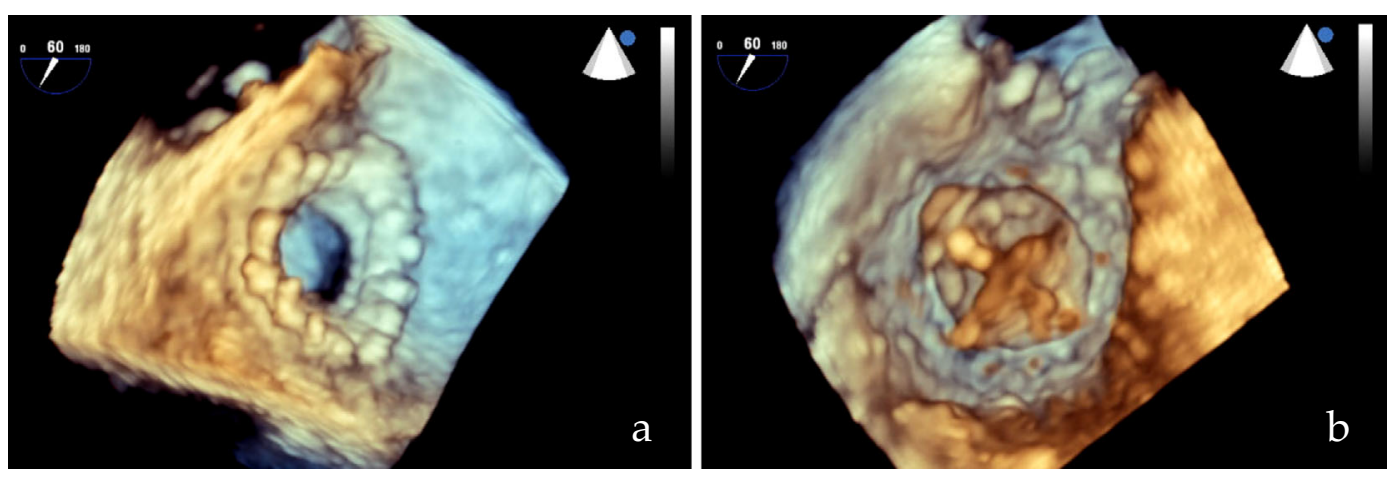

Figure 3. Three-dimensional transesophageal echocardiography (3D TEE). 3D TEE did not show any abnormal structures that could cause mitral valve dysfunction. a: Atrial aspect. b: Ventricular aspect.
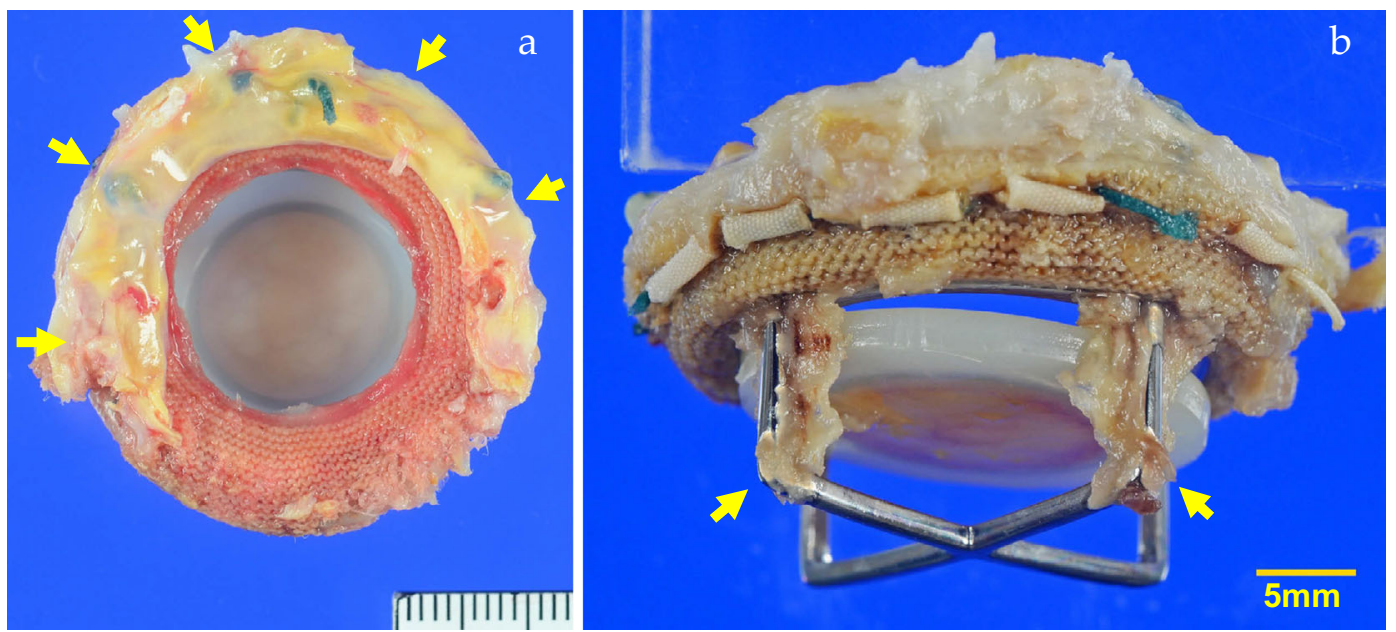

Figure 4. Macroscopic findings of the removed Starr-Edwards caged-disc. a: Pannus formation around the prosthetic valve annulus on the atrial side. Note the mild extension of the fibrinous tissue over the ring (yellow arrows). The lower part of the pannus in this figure was removed from the valve during the operation, which was comparable amount to the remaining tissues. b: Ventricular side of the frame and disc. Fibrinous soft tissue was attached to the frame (yellow arrows).

ventricular side (Fig. 4). After the operation, the patient's symptoms improved. Echocardiography showed the mean pressure gradient of the mitral valve to be $4 \mathrm{mmHg}$. He was discharged 11 days after the operation.

A pathologic examination of the removed valve revealed a mild extension of proliferated fibrinous tissue with calcification, or pannus, over the mitral annulus, as well as some soft fibrinous substance which was attached to the frame of the cage (Fig. 5).

\section{Discussion}

In this paper, we reported mitral valve stenosis of the SE caged-disc valve 35 years after the disc was replaced due to an abnormal soft fibrinous substance which was attached to the cage of the valve and mild pannus formation on the atrial aspect. To the best of our knowledge, this is the first report describing prosthetic valve failure with this kind of mechanism.
The SE caged-disc valve is a low-profile prosthetic valve developed to overcome the limitations of SE caged-ball valve. It began to be used in the 1970s, especially for patients whose ventricular chambers were small. This valve's use was discontinued after a short period of time because it wore out fairly quickly and caused thrombosis due to blood stream turbulence due to its structure (2). However, some patients have survived with this valve. The long-term outcomes of the SE caged-disc valve had not been fully investigated. Aoyagi et al. reported four patients who underwent MVR with the SE caged-disc valve over 30 years ago (1). The report described fracture, wear, and cracks of the SE caged-disc valve over 30 years after implantation. Pannus overgrowth after the implantation of SE caged-disc valve has also been previously reported (3).

There have also been a few reports regarding the hemodynamic evaluation of SE caged-disk valve without valve dysfunction, and the normal mean mitral diastolic gradient of the SE caged-disc valve was reported to range from 4.0 to 

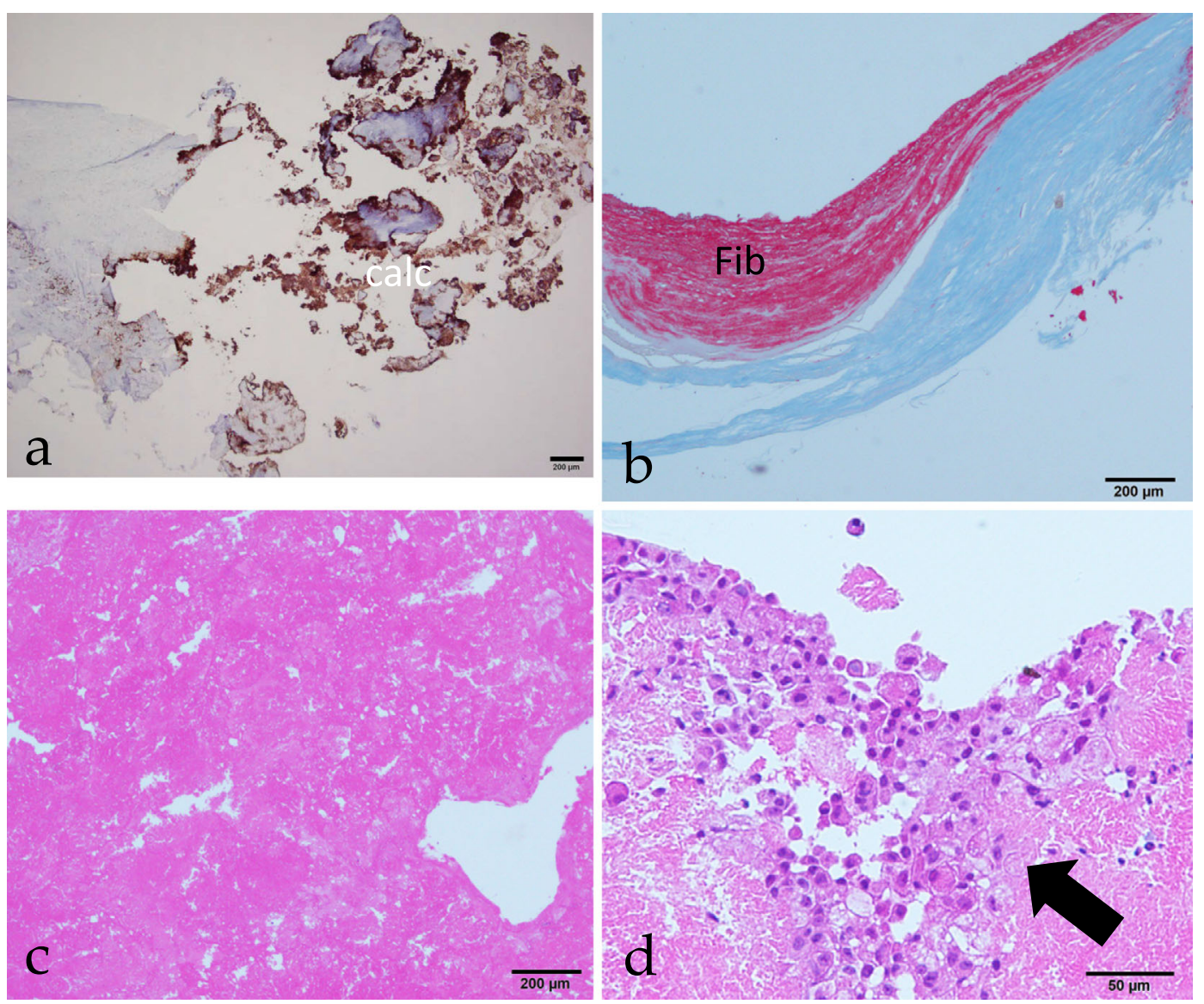

Figure 5. Histopathological findings of pannus and fibrinous substance. a: Immunostaining for osteopontin. Calcification is immunohistochemically positive for osteopontin (Leica, New Castle, UK, dilution 1:500). b: Masson's Trichrome staining. c, d: Hematoxylin and Eosin staining. calc: calcification, fib: fibrin. a: Granular calcified clusters are in the collagenous fiber of the pannus. b: Fibrinous tissue is also attached to the surface of the pannus. c: Fibrinous substance attached to the cage. d: High magnification of Fig. 5c. Macrophages (marked by arrows) were observed with the fibrin components.

$7.9 \mathrm{mmHg}$ (4-6). In our case, the mean mitral diastolic gradient was $12.3 \mathrm{mmHg}$, significantly higher than the normal range.

The pre-operative examination did not detect the anatomical cause of the elevated trans-valvular pressure. Intraoperative observation showed pannus formation and a fibrinous substance surrounding the prosthetic valve. There are no other findings to cause valve dysfunction, so we find it reasonable to assume that those factors caused prosthetic mitral valve stenosis. Evaluating a prosthetic mechanical valve via echocardiography is difficult. No motion abnormalities of the disc appeared on fluoroscopy. The soft fibrinous substance on the cage and pannus might have disrupted the blood flow through the prosthetic mitral valve without interfering with the disc's movement.

Persistent mechanical stress by a foreign object and exposure to a synthetic material can cause the human body to react to the item with macrophage infiltration. In turn, this might have caused pannus formation and fibrinous substance attachment long after the MVR procedure.

We herein reported a case of mitral valve stenosis due to a fibrinous substance attached to the cage and pannnus beneath the prosthetic valve. This situation occurred 35 years after MVR with a SE caged-disc valve. As a result, the condition was difficult to diagnose preoperatively. Patients who have a SE caged-disc valve need a careful long-term followup to identify any prosthetic valve dysfunction. If a patient with a caged valve showed an elevated trans-valvular gradient and no relevant structural abnormality, the possibility of fibrinous attachment to the cage of the valve and pannus formation should be suspected.

The authors state that they have no Conflict of Interest (COI).

\section{References}

1. Aoyagi S, Tayama K, Okazaki T, et al. Structural valve deterioration in a starr-edwards mitral caged-disk valve prosthesis. Circ J 77: 105-108, 2013.

2. Sakamoto Y, Hashimoto K. Development of prosthetic valves. The Journal of Adult Diseases 44: 859-864, 2014.

3. Aoyagi S, Fukunaga S, Tayama E, Arinaga K, Oda T, Kosuga T. 
Long-term survivors after valve replacement with a Starr-Edwards mitral disk valve prosthesis. Artif Organs 30: 484-487, 2006.

4. Brown JW, Myerowitz PD, Cann MS, Colvin SB, McIntosh CL, Morrow AG. Clinical and hemodynamic comparisons of KayShiley, Starr-Edwards No. 6520, and Reis-Hancock porcine xenograft mitral valves. Surgery 76: 983-991, 1974.

5. Nakano S, Mori T, Ohyama C, et al. [Late results of mitral valve replacement with Starr-Edwards low profile disc valve]. Kyobu Geka (The Japanese Journal of Thoracic Surgery) 31: 440-445, 1978 (in Japanese).

6. Kremau EL, Kloster FE, Griswold HE, Starr A. Hemodynamic evaluation of Starr-Edwards model 6520 mitral-tricuspid disc prosthesis. Circulation 178 (Suppl 2): 145-146, 1972.

(C) 2016 The Japanese Society of Internal Medicine http://www.naika.or.jp/imonline/index.html 